\title{
Respiration Rate Monitoring Methods: A Review
}

\begin{tabular}{|c|c|}
\hline Journal: & Pediatric Pulmonology \\
\hline Manuscript ID: & PPUL-10-0195.R2 \\
\hline Wiley - Manuscript type: & State of the Art \\
\hline $\begin{array}{r}\text { Date Submitted by the } \\
\text { Author: }\end{array}$ & 19-Nov-2010 \\
\hline Complete List of Authors: & $\begin{array}{l}\text { Al-Khalidi, Farah; Sheffield Hallam University, Faculty of ACES } \\
\text { Saatchi, Reza; Sheffield Hallam University, Faculty of ACES } \\
\text { Burke, Derek; Sheffield Children's Hospital, Emergency Department } \\
\text { Elphick, Heather; Sheffield Children's Hospital } \\
\text { Tan, Stephen; Sheffield Hallam University, Faculty of ACES }\end{array}$ \\
\hline Keywords: & respiratory, thermal imaging, non-contact \\
\hline
\end{tabular}




\section{Respiration Rate Monitoring Methods: A Review}

\section{F.Q. AL-Khalidi ${ }^{1}$, R. Saatchi ${ }^{1}$, D. Burke ${ }^{2}$, H. Elphick ${ }^{2}$ and S. Tan ${ }^{1}$}

${ }^{1}$ Faculty of ACES, Sheffield Hallam University, Sheffield, U.K.

${ }^{2}$ Sheffield Children's NHS Foundation Trust, Sheffield, U.K.

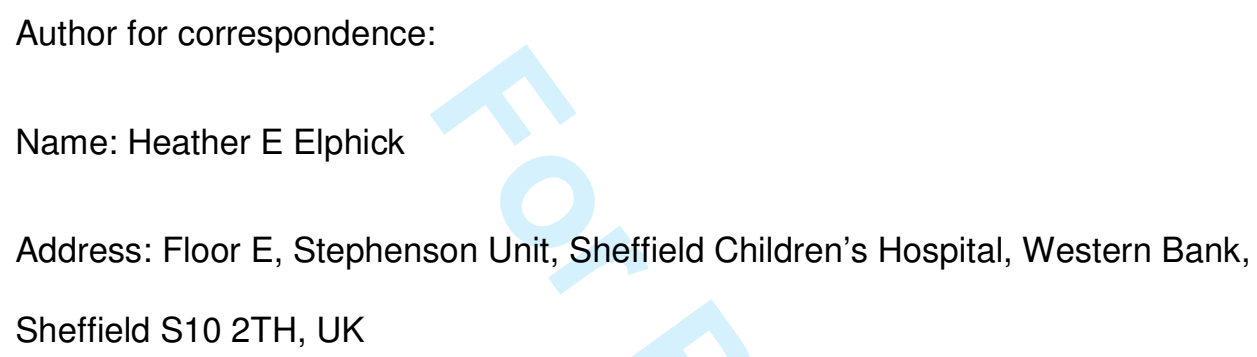

Email: Heather.Elphick@sch.nhs.uk

Short Title: Respiration Rate Monitoring Methods 


\section{Summary}

Respiration rate is an important indicator of a person's health, and thus it is monitored when performing clinical evaluations. There are different approaches for respiration monitoring, but generally they can be classed as contact or noncontact. For contact methods, the sensing device (or part of the instrument containing it) is attached to the subject's body. For noncontact approaches the monitoring is performed by an instrument that does not make any contact with the subject.

In this paper a review of respiration monitoring approaches (both contact and noncontact) is provided. Concerns related to the patient's recording comfort, recording hygiene, and the accuracy of respiration rate monitoring have resulted in the development of a number of noncontact respiration monitoring approaches.

A description of thermal imaging based and vision based noncontact respiration monitoring approaches we are currently developing is provided.

Keywords: Respiratory, thermal imaging, noncontact 


\section{Introduction}

Breathing is an important physiological task in living organisms. For humans, this process results in air containing oxygen being inhaled into the lungs, where gas exchange occurs across the alveolar-capillary membrane (1). Carbon Dioxide is excreted as part of the process, in the air released through the nose or mouth. The entire process from the inhalation to exhalation is known as a breathing (or respiration) cycle.

Respiratory rate is a vital sign used to monitor the progression of illness and an abnormal respiratory rate is an important marker of serious illness. There is substantial evidence that alterations in respiratory rate can be used to predict potentially serious clinical events such as cardiac arrest or admission to the intensive care unit (2-5). These studies have shown respiratory rate to be better than other vital measurements such as pulse and blood pressure in discriminating between stable patients and patient at risk (3). Using changes in respiratory rate measurements patients could have been identified as high risk up to 24 hours before the event with a specificity of $95 \%$ (5). Hospital systems such as the Paediatric Early Warning System (PEWS) have been developed to encourage appropriate responses to abnormal vital signs including an elevated respiratory rate.

Evidence from a recent audit of febrile children in our unit suggests that in both the emergency department and the acute medical admissions unit, respiratory rate was only measured in around two thirds of children on arrival. Reasons for omission were given as "crying" or "unsettled" and in many of these children experienced nursing 
staff were unable to obtain an accurate reading. However, it is in these children that this measurement would potentially be most useful and important.

According to Murthy (6), existing devices for monitoring respiratory rate only estimate the actual breathing rate due to their limitations. These devices can be classified in different ways, depending on the manner in which they operate and how they are used. In this paper, respiration rate monitoring devices are grouped as either contact or noncontact. In contact respiration rate monitoring, the instrument makes direct contact with the subject's body. However, in noncontact monitoring, the respiration rate is measured without the instrument making contact with the subject's body. There are clear advantages to noncontact respiration monitoring methods. These include improved patient comfort (especially for long term monitoring) as the subject is not tied to an instrument and improved accuracy as distress caused by a contact device may alter the respiration rate.

There have been several studies reporting developments in both contact and noncontact respiration monitoring. A review of non-invasive respiratory monitoring in medical care was provided by Folke et al. (7). This paper aims to review the literature on contact and non-contact methods of respiratory rate monitoring, including two methods currently being developed by our unit.

\section{Contact Based Respiration Monitoring}

Contact respiration rate monitoring instruments are usually based on measuring one of the following parameters: respiratory sounds, respiratory airflow, respiratory 


\section{1 $\quad$ Acoustic Based Methods}

Respiratory sound can be measured using a microphone placed either close to the respiratory airways or over the throat to detect the variation of sound. Then a frequency analysis and estimation of the loudness of the sound can be carried out (8).

Werthammer et al. (9) reported a respiratory sounds measurement system to detect sleep apnea in infants. The system depended on recording a signal derived from breathing sounds from the nose. This method was applied to eight premature infants. Snorting, speaking, crying, coughing etc had a negative effect on the operation of the system.

Corbishley and Rordriguez-Villegas (10) proposed a miniaturised and wearable respiration monitoring respiration system. It used a microphone mounted on the neck to obtain the largest breathing acoustic.

\subsection{Airflow Based Methods}

Airflow can be detected because exhaled air is warmer, has higher humidity and contains more $\mathrm{CO}_{2}$ than inhaled air. These variations can be used for indicating the respiratory rate. Most airflow-sensing methods need a sensor, attached to the airways (7). The measurement of the airflow can be achieved by using a nasal or oronasal thermistor which detects changes in temperature between the inhaled and 
exhaled air. This gives a semi-quantitative estimate of airflow, but the method is limited due to a high incidence of thermistor displacement (11).

The nasal pressure transducer is another sensor used to measure respiration rate. Nasal pressure is a more accurate measure of airflow than others as it based on the actual volume of the air exhaled $(8,12)$. It can be measured via nasal cannulae, mouthpiece or facemask. A problem with airflow measurement is that some patients may not feel comfortable with the sensor (13). The collector can also affect respiratory activity by increasing deadspace.

Folke et al. (14) have reported a $\mathrm{CO}_{2}$ sensor to measure respiration rate. Their study also indicated that subtle design changes in the collecting device could introduce large differences in sensor performance.

\subsection{Chest and Abdominal Movement Detection}

Chest and abdominal wall movements can best be measured by either mercury strain gauges or impedance methods. Respiratory inductance plethysmography is a non-invasive technique whereby two bands measure the respiration rate, the thoracic band which is placed around the rib cage and the abdominal band which is placed over the abdomen at the level of the umbilicus. The bands are made from an extendible/deformable conducting material, either a very fine wire or thin foil such that the conductivity can be maintained during the stretching process $(12,15)$. The principle of the strain gauge sensor is based on increase in the resistance of a conductor when the area of the conductor is increased during the respiration process. 
1

Normally the inspiratory thoracic and abdominal expansion is almost synchronous. However, if the upper airway is partially obstructed, there may be a change in the phase angle and timing of the movements of the thorax and abdomen (16). The movements become asynchronous, ie the thorax moves inwards, and the abdomen outwards. During expiration this pattern is then reversed. Thoraco-abdominal asynchrony is a normal finding in infants in whom chest wall compliance is greater (17) and is exacerbated by respiratory disease or respiratory muscle weakness (18). Nepal et al. (19) studied the abdominal strain gauge transducer for measuring respiration rate. The strain gauge was strapped around the patient's chest and changes in thoracic or abdominal circumference during breathing were measured. The method involved a classification algorithm to separate respiratory signals as apnoea, respiration, or respiration with motion, by using a second order autoregressive modelling and zero cross algorithm.

\subsection{Transcutaneous $\mathrm{CO}_{2}$ Monitoring}

In transcutaneous $\mathrm{CO}_{2}$ monitoring a heated electrode (about $42^{\circ} \mathrm{C}$ ) is applied to the skin (usually an arm). This method relies on the diffusion of gas to the skin and provides an overall estimate of change in $\mathrm{CO}_{2}$ level rather than minute by minute readings. The electrode is surrounded by a solution to provide conductivity. Care needs to be taken to avoid skin burning on sensitive and neonatal skin (12). Transcutaneous $\mathrm{CO}_{2}$ monitoring therefore allows measurements of consequences of abnormal ventilation rather than a measure of the respiratory rate itself. 


\subsection{Oximetry Probe $\left(\mathrm{SpO}_{2}\right)$ Based}

Blood-Oxygen saturation $\left(\mathrm{SpO}_{2}\right)$ measurement is another technique for monitoring the consequences of abnormal ventilation. When air enters the lungs its oxygen binds to the haemoglobin in red blood cells. The oxygen is then transported throughout the body in arterial blood. A pulse oximeter uses the red and infrared frequencies to determine the percentage of haemoglobin in the blood that is saturated with oxygen. This percentage is called blood saturation, or $\mathrm{SpO}_{2}(20)$. An oximeter simultaneously displays the $\mathrm{SpO}_{2}$ level as well as the pulse rate and plethysmogram.

There have been studies indicating that respiration rate can be extracted from plethysmograms. Plethysmograms from ten healthy adults were processed using wavelet transforms (21). Respiration waveform was observed in the plots of the wavelet transforms. In another study involving 14 infants, of median age 2 days, the feasibility of extracting respiratory information from the plethsmogram traces was also demonstrated (22). The magnitude frequency spectra of the plethsmogram traces showed peaks associated with respiration rate.

\subsection{Electrocardiogram (ECG) Derived Respiration Rate}

This method is based on the fact that respiration has a modulating effect on the ECG. In this respiration rate monitoring approach, ECG electrodes are attached to the subject in order to record an ECG. By measuring the fluctuation in ECG, the respiration rate can be derived. This technique is called ECG-Derived Respiration (EDR) and is based on a process known as sinus arrhythmia, i.e. the modulation of ECG by the breathing process (23). 
1

EDR is believed to be based on small ECG morphology changes during the respiratory cycle caused by movement of the heart position relative to the electrodes and the change in lung volume. Principal component analysis has been used to identify which ECG lead was most effective before extracting the respiration rate (24).

EDR monitoring has also been performed by using a single-channel that did not have to be a precordial lead (25). In contrast to a number of other studies that used ECG characteristic waves (e.g. QRS complex), this study used the higher order statistics of ECG recording (such as the 4th order cumulant).

A study carried out a quantitative comparison of EDR monitoring techniques based on direct measurement of the modulation components versus techniques based on calculation of the mean electrical axis variation (26). The study concluded that single lead respiration rate estimates are more robust than the methods based on the mean electrical axis.

Tarassenko et al. (27) looked at three methods: The EDR, the electrical impedance pneumography (IP) signal across the chest, and the ECG or the changes in light absorption which is known as photoplethysmogram (PPG) across a finger. They obtained estimates of the breathing rate by adding the individual estimated outputs for both the IP and PPG channels after applying the Kalman filters for both waveforms. A limitation of these methods is that movement artefacts introduce errors in estimating the breathing rates. 


\section{Noncontact Respiratory Monitoring Methods}

\subsection{Radar Based Respiration Rate Monitoring}

Greneker (28) reported one of the first noncontact respiration rate monitoring systems. The system was called the Radar Vital Signs Monitor (RVSM). It was developed to monitor the performance of Olympic athletes at distances exceeding 10 meters. The RVSM detected breathing-induced movements of the chest using the Doppler phenomenon. A limitation of this method was motion artefact which corrupted the breathing signals. There have been no published studies describing the use of this method in children.

\subsection{Optical Based Respiration Rate Monitoring}

Aoki et al. (29) reported a non-restrictive visual sensing method to detect the respiration pattern by using a Fiber Grating ( $F G$ ) vision sensor and processor unit. Their system consisted of two parts. The first was the Fibre Grating projecting device. This provided an array of invisible infra-red light spots (wavelength 810nm). The second part was a Charge-Coupled Device (CCD) camera with an optical bandpass filter. Infrared light was used to project a set of bright spots on the subject, while the CCD camera was used to capture the scene of bright spots. The moving distances of bright spots in each image were extracted and analysed to monitor respiration.

Nakajima et al. (30) used a static camera to detect thoracic movements to determine respiration rate. The projection of the surface of the thorax was represented as a region with a range of brightness intensities. Respiration was monitored by quantifying the variations of the locations of the image intensities over time. 
Non-invasive optical methods have not previously been described in children, however, in a study carried out by our group, a high speed desktop computer connected to a video camera (webcam) was used to record respiration related chest and abdominal movements in children in a sleep unit (31). The video consisted of a series of sequential images, each marked with an individual time stamp $(t)$, corresponding to the time of the recording. An algorithm was designed to subtract the current image at time $t$ (i.e. $i m g_{t}$ ) and the image a few time frames before at time $t$-td (i.e. $i m g_{t-t d}$ ). The value of $t d$ was determined practically. An increase in the value of $t d$ caused faster algorithm operation, but decreased the resolution of the algorithm in detecting movements. In this study a value of 0.5 second was used for $t d$ as it was observed to provide an acceptable compromise between the resolution and speed. The resulting difference images ( $i m g_{\text {diff }}$ ) were then thresholded to produce binary images $\left(i m g_{x}\right)$. The sum of pixel values in each binary image was determined to produce a set of data values $(x)$. The magnitude of each data value in $x$ represented the movement between the time $t$ and $t$-td. These values were then plotted against time to obtain a respiration signal.

A plot of the values of $x$ during exhalation and inhalation is provided in Fig.1. The vertical axis of the plot represents chest and abdominal movements. Two lobes were observed, the lobe representing inhalation being larger. During exhalation, the chest's wall initially moves slowly inward, its movement increases with time, reaching a peak and then, the amount of movement decreases. A similar process occurs during inhalation, but this time the chest's wall moves outward. Fig. 2 shows the plot of $x(t)$ during three respiration cycles. An algorithm was developed to extract the respiration rate from the recorded signal in real-time. 


\subsection{Thermal Sensor and Thermal Imaging Based Respiration Rate Monitoring}

Hsu and Chow (32) have reported a thermal sensor based respiration rate monitoring system. In this approach there was no contact with the child's skin. The sensor could detect temperature changes induced by respiration and then the data were corrected and analysed simultaneously by a personal computer that was linked to a central nursery room. To avoid missing the breathing signals, an ellipsoidshaped mask was made and the thermo sensors were placed on the mask so that breathing could be detected when the child's head turned. The problem with this method was that a mask had to be placed close to the child's face.

Zhu et al. (33) developed an infrared imaging based respiration rate monitoring method. They designed a tracking algorithm that could follow facial features related to respiration. These features were selected manually from a reference image (i.e. the first image in the video) by specifying three windows. Two of these windows covered the areas between the bridge of nose and the inner corner of the eyes (i.e. the periorbital regions) and represented the warmest facial areas. Another window was placed on the apex of nose to represent the coolest facial area. Their algorithm tracked these three windows in the following recorded images. The respiration signal was obtained from a rectangular region under the nose.

Instead of using a focal plane array of mid-wave infra-red sensors, Chekmenev, et al. (34) used a thermal camera consisting of a focal plane array for a long-wave infra-red $(6-15 \mu \mathrm{m})$ sensor. They measured the temperature changes around the neck region, carotid vessel complex, and the nasal region. The selection of these regions was carried out manually. A wavelet analysis technique was developed to extract the ECG and the respiration rate. 
1

In a study carried out by our group, the FLIR A40 thermal camera was used to monitor respiration related skin surface temperature variation in area centred on the tip of the nose (35). The thermal sensitivity of the camera is 0.08 Kelvin. The camera was fixed on a tripod in front of the subject at a distance of about one meter. The image capture rate was 50 frames per second, thus providing 3000 images per minute. The recording time was two minutes. The images were segmented and then an algorithm was used to locate and track a circular area centred on the tip of the nose. The segmentation and tracking algorithms are described Al-Khalidi et al. (36). The chosen area was divided into eight equal concentric segments as shown in Fig.3. The pixel values within each of the eight segments were averaged to obtain a single value representing the skin temperature in that segment. The process was repeated for each image. Plots of the average temperature against time for the segments were obtained (see Fig.4). These plots represented the respiration signal associated with the segments. The respiration signal reduces in amplitude during inhalation and increases in amplitude during exhalation. The clarity of the signals varied, with segments 3 and 7 providing the most clear signals and segment 1 providing the least clear signal. This result indicated that for respiration monitoring, it would be advisable to consider all the eight segments and then to select the segments that provide the clearest respiration signal. An algorithm was produced to automatically extract the respiration rate from the recorded signal.

\section{Discussion}

Respiration rate monitoring has evolved from the days when it could only be measured by placing the back of the hand close to the nose to monitor exhaled air. It 
is very important to develop a reliable, accurate and objective method of monitoring respiratory rate in children which is non-invasive and therefore will not cause distress to the child which may lead to inaccuracies in the measurement. The gold standard is still to place a thermistor in a nostril to monitor exhaled air. However, a variety of contact and noncontact respiration monitoring devices have been developed

Several techniques are available for measurement of respiratory rate via nasal

prongs, masks, thermistor or respiratory impedance plethysmography. These

methods all involve some contact with the child. A pneumotachograph has the

advantage that it gives quantitative assessments of flows, from which respiratory rate can be derived, and may be important in a research setting. The thermistor is a commonly used technique to detect temperature changes breath by breath at the nostril, thereby giving the number of breaths per minute. This technique has been formally validated in the measurement of respiratory rate for the purposes of sleep studies (11). A semi-quantitative measure of airflow and tidal volume can be derived from respiratory inductance plethysmography (RIP). The technique has been demonstrated to work well in measuring tidal volume in infants (37).

However, from a clinical perspective the main disadvantage of these methods is that they require connection to the child, some to the facial area, which disturbs many children and may be poorly tolerated. In addition, in infants and children, the added dead space of the equipment required for some of these techniques may have an influence on breathing patterns (38).

There are extensive opportunities to further improve noncontact respiration monitoring schemes. Radar, infrared imaging and optical imaging have all been used to monitor respiration rate. Advances in computing technology have resulted in 
1

realisation of complex algorithms for respiration monitoring. For example, it has been possible to use 3-dimentional vision algorithms and two web cameras to monitor respiration in manikins (36).

Currently noncontact respiration monitoring methods have not yet reached the level of maturity that can be used routinely in clinical environments. Concerns related to patient safety, electromagnetic interference with existing medical equipments, complexity of using the systems have been factors in their slow take up in clinical environments. With further development, noncontact methods will gradually become more and more viable and will effectively complement contact based respiration rate monitoring methods.

We have developed two novel methods for non contact respiratory rate monitoring in children which, whilst currently are sophisticated, high technology devices are undergoing further evaluation in acute clinical settings and will serve as a basis for future development to produce an inexpensive, user friendly device for use in the clinical environment.

\section{Conclusions}

Monitoring respiration rate is an important task when evaluating a subject's health.

Respiration rate monitoring devices can be classified by a number of ways depending on the manner of their use and their operation. In this review paper, they were grouped into contact and noncontact. This review has highlighted the advances made to improve the effectiveness of respiration monitoring. The potential for noncontact respiration monitoring is emphasised. Noncontact respiration rate 
monitoring devices have a distinct advantage over contact methods, especially in children, as they cause least disturbance to the patient. Studies are still ongoing to produce more effective respiration monitoring devices. 


\section{References}

1. Lausted CG, Jonston AT. Respiratory system. In: Bronzino JD, editor. Biomedical engineering fundamentals. CRC Press 2006.

2. Fieselmann JF, Hendryx MS, Helms $\mathrm{CM}$, et al. Respiratory rate predicts cardiopulmonary arrest for internal medicine patients. J Gen Intern Med 1993;8:354-360

3. Subbe CP, Davies RG, Williams E, et al. Effect of introducing the Modified Early Warning score on clinical outcomes, cardiopulmonary arrests and intensive care utilisation in acute medical admissions. Anaesthesia 2003;58:797-802

4. Goldhill DR, McNarry AF, Manersloot G, et al. A physiologically-based early warning score for ward patients: the association between score and outcome. Anaesthesia 2005;60:547-553

5. Cretikos M, Chen J, Hillman K, et al. The Objective Medical Emergency Team Activation Criteria: a case-control study. Resuscitation 2007;73:62-72

6. Murthy R, Pavlidis I, Tsiamyrtzis P. Touchless monitoring of breathing function. Engineering in Medicine and Biology Society, 2004. IEMBS apos;04. 26th Annual International Conference of the IEEE. Vol. 2, No. 1, pp(s):1196 - 1199.

7. Folke M, Cernerud L, Ekstro M, Hok B. Critical review of non-invasive respiratory monitoring in medical care. Medical and Biological Engineering and Computing. 2003; 41(4):377-383.

8. Shneerson MJ. Sleep Medicine: A Guide to Sleep and its Disorders, Handbook Second edition. Blackwell Publishing Ltd UK, 2005. 
9. Werthammer J, Krasner J, DiBenedetto J, Stark RA. Apnea Monitoring by Acoustic Detection of Airflow. Pediatrics .1983;71:53-55.

10. Corbishley P, Rodriguez-Villegas. Breathing detection: toward a miniaturised, wearable, battery-operated monitoring system. IEEE Transactions on Biomedical Engineering. 2008;.55 (1):196:204.

11. Storck K, Karlsson M, Ask P, Loyed D. Heat transfer evaluation of the nasal thermistor technique. IEEE Transactions on Biomedical Engineering 1996; 43 (12): 1187-1191.

12. Lee-Chiong LT, editor. Sleep: a comprehensive handbook. Wiley 2006.

13. Tobin MJ. Respiratory Monitoring in the Intensive Care Unit. Am Rev Repatriation Dis.1988; 138(6): 1625-1642.

14. Folke $M$, Granstedt $F$, Hok $B$ Scheer $H$. Comparative provocation test of Deleted: Cernerud L, Ekstro M, respiratory monitoring methods. Journal of Clinical Monitoring and Computing 2002;17(2):97-103.

15. Blom AJ. Monitoring of respiration and circulation 2004. CRC Press LLC, Boca Raton London New York Washington, D.C.

16. Prisk GK, Hammer J, Newth CJ. Techniques for measurement of thoracoabdominal asynchrony. Pediatr Pulmonol 2002 Dec;34(6):462-72.

17. Mayer OH, Clayton RG, Sr, Jawad AF, McDonough JM, Allen JL. Respiratory Inductance Plethysmography in Healthy 3- to 5-Year-Old Children. Chest. 2003 November 1, 2003;124(5):1812-9. 
1

18. Stromberg NO, Nelson N. Thoracoabdominal asynchrony in small children with lung disease--methodological aspects and the relationship to lung mechanics. Clin Physiol 1998 Sep;18(5):447-56.

19. Nepal K, Biegeleisen E, Ning T. Apnea Detection and Respiration rate Estimation through Parametric Modeling. Proceedings of the 28th IEEE Annual Northeast Bioengineering Conference, Philadelphia, Pennsylvania. 2002: 277-278.

20. Konica Minolta Sensing, Inc., Basic understanding of the pulse oximeter. 21. Leonard P, Beattie TF, Addison PS, Watson JN. Standard pulse oximeters can be used to monitor respiratory rate, Emerg Med Journal, 2003; 20:524-525.

22. Wertheim D, Olden C, Savage E, Seddon, P. Extracting respiratory data from pulse oximeter plethysmogram traces in newborn infants, Arch Dis Child Fetal Neonatal Ed, 2009; 94:301-303.

23. Moody G, Mark R, Bump M, Weinstein J, Berman A, Mietus J, Goldberger A. Clinical validation of ECG-derived respiration (EDR) technique. Computers in Cardiology, 1986;13:507- 510.

24. Mazzanti B, Lamberti C, de Bie J. Validation of an ECG-derived respiration monitoring method. Computers in Cardiology 2003;30:613-616.

25. Ding S, Zhu X, Chen W, Wei D. Derivation of respiratory signal from singlechannel ECGs based on source Ding statistics, International Journal of Bioelectromagnetism, 6(1):43-49.

26. O'Brien C, Heneghan C. Comparison of algorithms for estimation of respiratory signal from the surface electrocardiogram, Computers in Biology and Medicine, $2007 ; 37(3): 305-314$.

27. Tarassenko L, Mason L, Townsend N. Multi-sensor fusion for robust computation of breathing rate. IEEE Society, 2002; 38:1314-1316. 
28. Greneker EF. Radar sensing of heartbeat and respiration at a distance with applications of the technology. Radar 1997; 97:150:154.

29. Aoki H, Takemura Y, Mimura K, Nakajima M. Development of non-restrictive sensing system for sleeping person using fibre grating vision sensor. Proceedings of 2001 International Symposium on Micromechatronics and Human Science, Nagoya, Japan:155:160.

30. Nakajima K, Matsumoto Y, Tamura T. Development of real time image sequence analysis for evaluating posture change and respiratory rate of a subject in bed. Physiological Measurement 2001;22(3):21-28.

31. Tan KS, Saatchi R, Elphick H, Burke D. Real-time vision based respiration monitoring system. IEEE, IET International Symposium on Communication Systems, Networks and Digital Signal Processing 2010; 846-850 ISBN-Hardcopy: 988-186135-370-2

32. Hsu CH, Chow CJ. Design and clinic monitoring of monitoring of a newly developed non -attached infant apnea monitor. Centre for Biomedical Engineering, Taiwan, Province De Chine, 2005;17:126-133.

33. Zhu Z, Fei J, Pavlidis I. Tracking Human Breath in Infrared Imaging. Proceeding of the. 5th IEEE, Symposium on Bioinformatics and Bioengineering (BIBE'05). Organization IEEE Computer Society Washington, DC, USA. 2005; 19, Issue. 21: 227-231.

34. Chekmenev Y, Rara H, Farag A. Non-contact, wavelet-based measurement of vital signs using thermal imaging. ICGST International Journal on Graphics, Vision and Image Processing. 2006;6(2):25-30.

35. Saatchi R, AL-Khalidi F, Burke D, Elphick H. Thermal Image Analysis of the Skin 
Surface Centred on the Tip of the Nose for Respiration Monitoring. ICEDSP International Conference on Computer Systems and Applications, Manipal, India, 2009.

36. Al-Khalidi FQ, Saatchi R, Burke D, Elphick $H$. Facial tracking method for noncontact respiration rate monitoring, IEEE, IET International Symposium on Communication Systems, Networks and Digital Signal Processing, Newcastle, UK. 21-23 July, 2010; 827-830. ISBN-Hardcopy: 978-1-86135-370-2

37. Hershenson MB, Colin AA, Wohl ME, Stark AR. Changes in the contribution of the rib cage to tidal breathing during infancy. American Review of Respiratory

Disease. 1990;141:922-5.

38. Brooks LJ, DiFiore JM, Martin RJ. Assessment of tidal volume over time in preterm infants using respiratory inductance plethysmography, The CHIME Study Group. Collaborative Home Infant Monitoring Evaluation. Pediatr Pulmonol. 1997;23:429-33. 


\title{
Respiration Rate Monitoring Methods: A Review
}

\author{
F.Q. AL-Khalidi ${ }^{1}$, R. Saatchi ${ }^{1}$, D. Burke ${ }^{2}$, H. Elphick ${ }^{2}$ and S. Tan ${ }^{1}$ \\ ${ }^{1}$ Faculty of ACES, Sheffield Hallam University, Sheffield, U.K. \\ ${ }^{2}$ Sheffield Children's NHS Foundation Trust, Sheffield, U.K.
}

\begin{abstract}
Author for correspondence:
Name: Heather E Elphick

Address: Floor E, Stephenson Unit, Sheffield Children's Hospital, Western Bank, Sheffield S10 2TH, UK

Tel: (0)114 2717585

Email: Heather.Elphick@sch.nhs.uk
\end{abstract}

Short Title: Respiration Rate Monitoring Methods 


\section{Summary}

Respiration rate is an important indicator of a person's health, and thus it is monitored when performing clinical evaluations. There are different approaches for respiration monitoring, but generally they can be classed as contact or noncontact. For contact methods, the sensing device (or part of the instrument containing it) is attached to the subject's body. For noncontact approaches the monitoring is performed by an instrument that does not make any contact with the subject.

In this paper a review of respiration monitoring approaches (both contact and noncontact) is provided. Concerns related to the patient's recording comfort, recording hygiene, and the accuracy of respiration rate monitoring have resulted in the development of a number of noncontact respiration monitoring approaches.

A description of thermal imaging based and vision based noncontact respiration monitoring approaches we are currently developing is provided.

Keywords: Respiratory, thermal imaging, noncontact 


\section{Introduction}

Breathing is an important physiological task in living organisms. For humans, this process results in air containing oxygen being inhaled into the lungs, where gas exchange occurs across the alveolar-capillary membrane (1). Carbon Dioxide is excreted as part of the process, in the air released through the nose or mouth. The entire process from the inhalation to exhalation is known as a breathing (or respiration) cycle.

Respiratory rate is a vital sign used to monitor the progression of illness and an abnormal respiratory rate is an important marker of serious illness. There is substantial evidence that alterations in respiratory rate can be used to predict potentially serious clinical events such as cardiac arrest or admission to the intensive care unit (2-5). These studies have shown respiratory rate to be better than other vital measurements such as pulse and blood pressure in discriminating between stable patients and patient at risk (3). Using changes in respiratory rate measurements patients could have been identified as high risk up to 24 hours before the event with a specificity of $95 \%$ (5). Hospital systems such as the Paediatric Early Warning System (PEWS) have been developed to encourage appropriate responses to abnormal vital signs including an elevated respiratory rate.

Evidence from a recent audit of febrile children in our unit suggests that in both the emergency department and the acute medical admissions unit, respiratory rate was only measured in around two thirds of children on arrival. Reasons for omission were given as "crying" or "unsettled" and in many of these children experienced nursing 
staff were unable to obtain an accurate reading. However, it is in these children that this measurement would potentially be most useful and important.

According to Murthy (6), existing devices for monitoring respiratory rate only estimate the actual breathing rate due to their limitations. These devices can be classified in different ways, depending on the manner in which they operate and how they are used. In this paper, respiration rate monitoring devices are grouped as either contact or noncontact. In contact respiration rate monitoring, the instrument makes direct contact with the subject's body. However, in noncontact monitoring, the respiration rate is measured without the instrument making contact with the subject's body. There are clear advantages to noncontact respiration monitoring methods. These include improved patient comfort (especially for long term monitoring) as the subject is not tied to an instrument and improved accuracy as distress caused by a contact device may alter the respiration rate.

There have been several studies reporting developments in both contact and noncontact respiration monitoring. A review of non-invasive respiratory monitoring in medical care was provided by Folke et al. (7). This paper aims to review the literature on contact and non-contact methods of respiratory rate monitoring, including two methods currently being developed by our unit.

\section{Contact Based Respiration Monitoring}

Contact respiration rate monitoring instruments are usually based on measuring one of the following parameters: respiratory sounds, respiratory airflow, respiratory 
related chest or abdominal movements, respiratory $\mathrm{CO}_{2}$ emission and oximetry probe $\mathrm{SpO} 2$. Respiration rate can also be derived from the electrocardiogram (ECG).

\subsection{Acoustic Based Methods}

Respiratory sound can be measured using a microphone placed either close to the respiratory airways or over the throat to detect the variation of sound. Then a frequency analysis and estimation of the loudness of the sound can be carried out (8).

Werthammer et al. (9) reported a respiratory sounds measurement system to detect sleep apnea in infants. The system depended on recording a signal derived from breathing sounds from the nose. This method was applied to eight premature infants. Snorting, speaking, crying, coughing etc had a negative effect on the operation of the system.

Corbishley and Rordriguez-Villegas (10) proposed a miniaturised and wearable respiration monitoring respiration system. It used a microphone mounted on the neck to obtain the largest breathing acoustic.

\subsection{Airflow Based Methods}

Airflow can be detected because exhaled air is warmer, has higher humidity and contains more $\mathrm{CO}_{2}$ than inhaled air. These variations can be used for indicating the respiratory rate. Most airflow-sensing methods need a sensor, attached to the airways (7). The measurement of the airflow can be achieved by using a nasal or oronasal thermistor which detects changes in temperature between the inhaled and 
exhaled air. This gives a semi-quantitative estimate of airflow, but the method is limited due to a high incidence of thermistor displacement (11).

The nasal pressure transducer is another sensor used to measure respiration rate. Nasal pressure is a more accurate measure of airflow than others as it based on the actual volume of the air exhaled $(8,12)$. It can be measured via nasal cannulae, mouthpiece or facemask. A problem with airflow measurement is that some patients may not feel comfortable with the sensor (13). The collector can also affect respiratory activity by increasing deadspace.

Folke et al. (14) have reported a $\mathrm{CO}_{2}$ sensor to measure respiration rate. Their study also indicated that subtle design changes in the collecting device could introduce large differences in sensor performance.

\subsection{Chest and Abdominal Movement Detection}

Chest and abdominal wall movements can best be measured by either mercury strain gauges or impedance methods. Respiratory inductance plethysmography is a non-invasive technique whereby two bands measure the respiration rate, the thoracic band which is placed around the rib cage and the abdominal band which is placed over the abdomen at the level of the umbilicus. The bands are made from an extendible/deformable conducting material, either a very fine wire or thin foil such that the conductivity can be maintained during the stretching process $(12,15)$. The principle of the strain gauge sensor is based on increase in the resistance of a conductor when the area of the conductor is increased during the respiration process. 
Normally the inspiratory thoracic and abdominal expansion is almost synchronous. However, if the upper airway is partially obstructed, there may be a change in the phase angle and timing of the movements of the thorax and abdomen (16). The movements become asynchronous, ie the thorax moves inwards, and the abdomen outwards. During expiration this pattern is then reversed. Thoraco-abdominal asynchrony is a normal finding in infants in whom chest wall compliance is greater (17) and is exacerbated by respiratory disease or respiratory muscle weakness (18).

Nepal et al. (19) studied the abdominal strain gauge transducer for measuring respiration rate. The strain gauge was strapped around the patient's chest and changes in thoracic or abdominal circumference during breathing were measured. The method involved a classification algorithm to separate respiratory signals as apnoea, respiration, or respiration with motion, by using a second order autoregressive modelling and zero cross algorithm.

\subsection{Transcutaneous $\mathrm{CO}_{2}$ Monitoring}

In transcutaneous $\mathrm{CO}_{2}$ monitoring a heated electrode (about $42^{\circ} \mathrm{C}$ ) is applied to the skin (usually an arm). This method relies on the diffusion of gas to the skin and provides an overall estimate of change in $\mathrm{CO}_{2}$ level rather than minute by minute readings. The electrode is surrounded by a solution to provide conductivity. Care needs to be taken to avoid skin burning on sensitive and neonatal skin (12). Transcutaneous $\mathrm{CO}_{2}$ monitoring therefore allows measurements of consequences of abnormal ventilation rather than a measure of the respiratory rate itself. 


\subsection{Oximetry Probe $\left(\mathrm{SpO}_{2}\right)$ Based}

Blood-Oxygen saturation $\left(\mathrm{SpO}_{2}\right)$ measurement is another technique for monitoring the consequences of abnormal ventilation. When air enters the lungs its oxygen binds to the haemoglobin in red blood cells. The oxygen is then transported throughout the body in arterial blood. A pulse oximeter uses the red and infrared frequencies to determine the percentage of haemoglobin in the blood that is saturated with oxygen. This percentage is called blood saturation, or $\mathrm{SpO}_{2}(20)$. An oximeter simultaneously displays the $\mathrm{SpO}_{2}$ level as well as the pulse rate and plethysmogram.

There have been studies indicating that respiration rate can be extracted from plethysmograms. Plethysmograms from ten healthy adults were processed using wavelet transforms (21). Respiration waveform was observed in the plots of the wavelet transforms. In another study involving 14 infants, of median age 2 days, the feasibility of extracting respiratory information from the plethsmogram traces was also demonstrated (22). The magnitude frequency spectra of the plethsmogram traces showed peaks associated with respiration rate.

\subsection{Electrocardiogram (ECG) Derived Respiration Rate}

This method is based on the fact that respiration has a modulating effect on the ECG. In this respiration rate monitoring approach, ECG electrodes are attached to the subject in order to record an ECG. By measuring the fluctuation in ECG, the respiration rate can be derived. This technique is called ECG-Derived Respiration (EDR) and is based on a process known as sinus arrhythmia, i.e. the modulation of ECG by the breathing process (23). 
EDR is believed to be based on small ECG morphology changes during the respiratory cycle caused by movement of the heart position relative to the electrodes and the change in lung volume. Principal component analysis has been used to identify which ECG lead was most effective before extracting the respiration rate (24).

EDR monitoring has also been performed by using a single-channel that did not have to be a precordial lead (25). In contrast to a number of other studies that used ECG characteristic waves (e.g. QRS complex), this study used the higher order statistics of ECG recording (such as the 4th order cumulant).

A study carried out a quantitative comparison of EDR monitoring techniques based on direct measurement of the modulation components versus techniques based on calculation of the mean electrical axis variation (26). The study concluded that single lead respiration rate estimates are more robust than the methods based on the mean electrical axis.

Tarassenko et al. (27) looked at three methods: The EDR, the electrical impedance pneumography (IP) signal across the chest, and the ECG or the changes in light absorption which is known as photoplethysmogram (PPG) across a finger. They obtained estimates of the breathing rate by adding the individual estimated outputs for both the IP and PPG channels after applying the Kalman filters for both waveforms. A limitation of these methods is that movement artefacts introduce errors in estimating the breathing rates. 


\section{Noncontact Respiratory Monitoring Methods}

\subsection{Radar Based Respiration Rate Monitoring}

Greneker (28) reported one of the first noncontact respiration rate monitoring systems. The system was called the Radar Vital Signs Monitor (RVSM). It was developed to monitor the performance of Olympic athletes at distances exceeding 10 meters. The RVSM detected breathing-induced movements of the chest using the Doppler phenomenon. A limitation of this method was motion artefact which corrupted the breathing signals. There have been no published studies describing the use of this method in children.

\subsection{Optical Based Respiration Rate Monitoring}

Aoki et al. (29) reported a non-restrictive visual sensing method to detect the respiration pattern by using a Fiber Grating (FG) vision sensor and processor unit. Their system consisted of two parts. The first was the Fibre Grating projecting device. This provided an array of invisible infra-red light spots (wavelength $810 \mathrm{~nm}$ ). The second part was a Charge-Coupled Device (CCD) camera with an optical bandpass filter. Infrared light was used to project a set of bright spots on the subject, while the CCD camera was used to capture the scene of bright spots. The moving distances of bright spots in each image were extracted and analysed to monitor respiration.

Nakajima et al. (30) used a static camera to detect thoracic movements to determine respiration rate. The projection of the surface of the thorax was represented as a region with a range of brightness intensities. Respiration was monitored by quantifying the variations of the locations of the image intensities over time. 
Non-invasive optical methods have not previously been described in children, however, in a study carried out by our group, a high speed desktop computer connected to a video camera (webcam) was used to record respiration related chest and abdominal movements in children in a sleep unit (31). The video consisted of a series of sequential images, each marked with an individual time stamp $(t)$, corresponding to the time of the recording. An algorithm was designed to subtract the current image at time $t$ (i.e. $i m g_{t}$ ) and the image a few time frames before at time $t$-td (i.e. $i m g_{t-t d)}$. The value of $t d$ was determined practically. An increase in the value of $t d$ caused faster algorithm operation, but decreased the resolution of the algorithm in detecting movements. In this study a value of 0.5 second was used for $t d$ as it was observed to provide an acceptable compromise between the resolution and speed. The resulting difference images $\left(i m g_{d i f f}\right)$ were then thresholded to produce binary images $\left(i m g_{x}\right)$. The sum of pixel values in each binary image was determined to produce a set of data values $(x)$. The magnitude of each data value in $x$ represented the movement between the time $t$ and $t$-td. These values were then plotted against time to obtain a respiration signal.

A plot of the values of $x$ during exhalation and inhalation is provided in Fig.1. The vertical axis of the plot represents chest and abdominal movements. Two lobes were observed, the lobe representing inhalation being larger. During exhalation, the chest's wall initially moves slowly inward, its movement increases with time, reaching a peak and then, the amount of movement decreases. A similar process occurs during inhalation, but this time the chest's wall moves outward. Fig. 2 shows the plot of $x(t)$ during three respiration cycles. An algorithm was developed to extract the respiration rate from the recorded signal in real-time. 


\subsection{Thermal Sensor and Thermal Imaging Based Respiration Rate Monitoring}

Hsu and Chow (32) have reported a thermal sensor based respiration rate monitoring system. In this approach there was no contact with the child's skin. The sensor could detect temperature changes induced by respiration and then the data were corrected and analysed simultaneously by a personal computer that was linked to a central nursery room. To avoid missing the breathing signals, an ellipsoidshaped mask was made and the thermo sensors were placed on the mask so that breathing could be detected when the child's head turned. The problem with this method was that a mask had to be placed close to the child's face.

Zhu et al. (33) developed an infrared imaging based respiration rate monitoring method. They designed a tracking algorithm that could follow facial features related to respiration. These features were selected manually from a reference image (i.e. the first image in the video) by specifying three windows. Two of these windows covered the areas between the bridge of nose and the inner corner of the eyes (i.e. the periorbital regions) and represented the warmest facial areas. Another window was placed on the apex of nose to represent the coolest facial area. Their algorithm tracked these three windows in the following recorded images. The respiration signal was obtained from a rectangular region under the nose.

Instead of using a focal plane array of mid-wave infra-red sensors, Chekmenev, et al. (34) used a thermal camera consisting of a focal plane array for a long-wave infra-red $(6-15 \mu \mathrm{m})$ sensor. They measured the temperature changes around the neck region, carotid vessel complex, and the nasal region. The selection of these regions was carried out manually. A wavelet analysis technique was developed to extract the ECG and the respiration rate. 
In a study carried out by our group, the FLIR A40 thermal camera was used to monitor respiration related skin surface temperature variation in area centred on the tip of the nose (35). The thermal sensitivity of the camera is 0.08 Kelvin. The camera was fixed on a tripod in front of the subject at a distance of about one meter. The image capture rate was 50 frames per second, thus providing 3000 images per minute. The recording time was two minutes. The images were segmented and then an algorithm was used to locate and track a circular area centred on the tip of the nose. The segmentation and tracking algorithms are described Al-Khalidi et al. (36). The chosen area was divided into eight equal concentric segments as shown in Fig.3. The pixel values within each of the eight segments were averaged to obtain a single value representing the skin temperature in that segment. The process was repeated for each image. Plots of the average temperature against time for the segments were obtained (see Fig.4). These plots represented the respiration signal associated with the segments. The respiration signal reduces in amplitude during inhalation and increases in amplitude during exhalation. The clarity of the signals varied, with segments 3 and 7 providing the most clear signals and segment 1 providing the least clear signal. This result indicated that for respiration monitoring, it would be advisable to consider all the eight segments and then to select the segments that provide the clearest respiration signal. An algorithm was produced to automatically extract the respiration rate from the recorded signal.

\section{Discussion}

Respiration rate monitoring has evolved from the days when it could only be measured by placing the back of the hand close to the nose to monitor exhaled air. It 
is very important to develop a reliable, accurate and objective method of monitoring respiratory rate in children which is non-invasive and therefore will not cause distress to the child which may lead to inaccuracies in the measurement. The gold standard is still to place a thermistor in a nostril to monitor exhaled air. However, a variety of contact and noncontact respiration monitoring devices have been developed.

Several techniques are available for measurement of respiratory rate via nasal prongs, masks, thermistor or respiratory impedance plethysmography. These methods all involve some contact with the child. A pneumotachograph has the advantage that it gives quantitative assessments of flows, from which respiratory rate can be derived, and may be important in a research setting. The thermistor is a commonly used technique to detect temperature changes breath by breath at the nostril, thereby giving the number of breaths per minute. This technique has been formally validated in the measurement of respiratory rate for the purposes of sleep studies (11). A semi-quantitative measure of airflow and tidal volume can be derived from respiratory inductance plethysmography (RIP). The technique has been demonstrated to work well in measuring tidal volume in infants (37).

However, from a clinical perspective the main disadvantage of these methods is that they require connection to the child, some to the facial area, which disturbs many children and may be poorly tolerated. In addition, in infants and children, the added dead space of the equipment required for some of these techniques may have an influence on breathing patterns (38).

There are extensive opportunities to further improve noncontact respiration monitoring schemes. Radar, infrared imaging and optical imaging have all been used to monitor respiration rate. Advances in computing technology have resulted in 
realisation of complex algorithms for respiration monitoring. For example, it has been possible to use 3-dimentional vision algorithms and two web cameras to monitor respiration in manikins (36).

Currently noncontact respiration monitoring methods have not yet reached the level of maturity that can be used routinely in clinical environments. Concerns related to patient safety, electromagnetic interference with existing medical equipments, complexity of using the systems have been factors in their slow take up in clinical environments. With further development, noncontact methods will gradually become more and more viable and will effectively complement contact based respiration rate monitoring methods.

We have developed two novel methods for non contact respiratory rate monitoring in children which, whilst currently are sophisticated, high technology devices are undergoing further evaluation in acute clinical settings and will serve as a basis for future development to produce an inexpensive, user friendly device for use in the clinical environment.

\section{Conclusions}

Monitoring respiration rate is an important task when evaluating a subject's health. Respiration rate monitoring devices can be classified by a number of ways depending on the manner of their use and their operation. In this review paper, they were grouped into contact and noncontact. This review has highlighted the advances made to improve the effectiveness of respiration monitoring. The potential for noncontact respiration monitoring is emphasised. Noncontact respiration rate 
monitoring devices have a distinct advantage over contact methods, especially in children, as they cause least disturbance to the patient. Studies are still ongoing to produce more effective respiration monitoring devices. 


\section{References}

1. Lausted CG, Jonston AT. Respiratory system. In: Bronzino JD, editor. Biomedical engineering fundamentals. CRC Press 2006.

2. Fieselmann JF, Hendryx MS, Helms CM, et al. Respiratory rate predicts cardiopulmonary arrest for internal medicine patients. J Gen Intern Med $1993 ; 8: 354-360$

3. Subbe CP, Davies RG, Williams E, et al. Effect of introducing the Modified Early Warning score on clinical outcomes, cardiopulmonary arrests and intensive care utilisation in acute medical admissions. Anaesthesia 2003;58:797-802

4. Goldhill DR, McNarry AF, Manersloot G, et al. A physiologically-based early warning score for ward patients: the association between score and outcome. Anaesthesia 2005;60:547-553

5. Cretikos M, Chen J, Hillman K, et al. The Objective Medical Emergency Team Activation Criteria: a case-control study. Resuscitation 2007;73:62-72

6. Murthy R, Pavlidis I, Tsiamyrtzis P. Touchless monitoring of breathing function. Engineering in Medicine and Biology Society, 2004. IEMBS apos;04. 26th Annual International Conference of the IEEE. Vol. 2, No. 1, pp(s):1196-1199.

7. Folke M, Cernerud L, Ekstro M, Hok B. Critical review of non-invasive respiratory monitoring in medical care. Medical and Biological Engineering and Computing. 2003; 41(4):377-383.

8. Shneerson MJ. Sleep Medicine: A Guide to Sleep and its Disorders, Handbook Second edition. Blackwell Publishing Ltd UK, 2005. 
9. Werthammer J, Krasner J, DiBenedetto J, Stark RA. Apnea Monitoring by Acoustic Detection of Airflow. Pediatrics .1983;71:53-55.

10. Corbishley P, Rodriguez-Villegas. Breathing detection: toward a miniaturised, wearable, battery-operated monitoring system. IEEE Transactions on Biomedical Engineering. 2008;.55 (1):196:204.

11. Storck K, Karlsson M, Ask P, Loyed D. Heat transfer evaluation of the nasal thermistor technique. IEEE Transactions on Biomedical Engineering 1996; 43 (12): 1187-1191.

12. Lee-Chiong LT, editor. Sleep: a comprehensive handbook. Wiley 2006.

13. Tobin MJ. Respiratory Monitoring in the Intensive Care Unit. Am Rev Repatriation Dis.1988; 138(6): 1625-1642.

14. Folke M, Granstedt F, Hok B, Scheer H. Comparative provocation test of respiratory monitoring methods. Journal of Clinical Monitoring and Computing $2002 ; 17(2): 97-103$.

15. Blom AJ. Monitoring of respiration and circulation 2004. CRC Press LLC, Boca Raton London New York Washington, D.C.

16. Prisk GK, Hammer J, Newth CJ. Techniques for measurement of thoracoabdominal asynchrony. Pediatr Pulmonol 2002 Dec;34(6):462-72.

17. Mayer $\mathrm{OH}$, Clayton RG, Sr, Jawad AF, McDonough JM, Allen JL. Respiratory Inductance Plethysmography in Healthy 3- to 5-Year-Old Children. Chest. 2003 November 1, 2003;124(5):1812-9. 
18. Stromberg NO, Nelson N. Thoracoabdominal asynchrony in small children with lung disease--methodological aspects and the relationship to lung mechanics. Clin Physiol 1998 Sep;18(5):447-56.

19. Nepal K, Biegeleisen E, Ning T. Apnea Detection and Respiration rate Estimation through Parametric Modeling. Proceedings of the 28th IEEE Annual Northeast Bioengineering Conference, Philadelphia, Pennsylvania. 2002: 277-278.

20. Konica Minolta Sensing, Inc., Basic understanding of the pulse oximeter.

21. Leonard P, Beattie TF, Addison PS, Watson JN. Standard pulse oximeters can be used to monitor respiratory rate, Emerg Med Journal, 2003; 20:524-525.

22. Wertheim D, Olden C, Savage E, Seddon, P. Extracting respiratory data from pulse oximeter plethysmogram traces in newborn infants, Arch Dis Child Fetal Neonatal Ed, 2009; 94:301-303.

23. Moody G, Mark R, Bump M, Weinstein J, Berman A, Mietus J, Goldberger A. Clinical validation of ECG-derived respiration (EDR) technique. Computers in Cardiology, 1986;13:507- 510.

24. Mazzanti B, Lamberti C, de Bie J. Validation of an ECG-derived respiration monitoring method. Computers in Cardiology 2003;30:613-616.

25. Ding S, Zhu X, Chen W, Wei D. Derivation of respiratory signal from singlechannel ECGs based on source Ding statistics, International Journal of Bioelectromagnetism, 6(1):43-49.

26. O'Brien C, Heneghan C. Comparison of algorithms for estimation of respiratory signal from the surface electrocardiogram, Computers in Biology and Medicine, 2007; 37(3):305-314.

27. Tarassenko L, Mason L, Townsend N. Multi-sensor fusion for robust computation of breathing rate. IEEE Society, 2002; 38:1314-1316. 
28. Greneker EF. Radar sensing of heartbeat and respiration at a distance with applications of the technology. Radar 1997; 97:150:154.

29. Aoki H, Takemura Y, Mimura K, Nakajima M. Development of non-restrictive sensing system for sleeping person using fibre grating vision sensor. Proceedings of 2001 International Symposium on Micromechatronics and Human Science, Nagoya, Japan:155:160.

30. Nakajima K, Matsumoto Y, Tamura T. Development of real time image sequence analysis for evaluating posture change and respiratory rate of a subject in bed. Physiological Measurement 2001;22(3):21-28.

31. Tan KS, Saatchi R, Elphick H, Burke D. Real-time vision based respiration monitoring system. IEEE, IET International Symposium on Communication Systems, Networks and Digital Signal Processing 2010; 846-850 ISBN-Hardcopy: 988-186135-370-2

32. Hsu $\mathrm{CH}$, Chow CJ. Design and clinic monitoring of monitoring of a newly developed non -attached infant apnea monitor. Centre for Biomedical Engineering, Taiwan, Province De Chine, 2005;17:126-133.

33. Zhu Z, Fei J, Pavlidis I. Tracking Human Breath in Infrared Imaging. Proceeding of the. 5th IEEE, Symposium on Bioinformatics and Bioengineering (BIBE’05). Organization IEEE Computer Society Washington, DC, USA. 2005; 19, Issue. 21: 227-231.

34. Chekmenev Y, Rara H, Farag A. Non-contact, wavelet-based measurement of vital signs using thermal imaging. ICGST International Journal on Graphics, Vision and Image Processing. 2006;6(2):25-30.

35. Saatchi R, AL-Khalidi F, Burke D, Elphick H. Thermal Image Analysis of the Skin 
Surface Centred on the Tip of the Nose for Respiration Monitoring. ICEDSP International Conference on Computer Systems and Applications, Manipal, India, 2009.

36. Al-Khalidi FQ, Saatchi R, Burke D, Elphick H. Facial tracking method for noncontact respiration rate monitoring, IEEE, IET International Symposium on Communication Systems, Networks and Digital Signal Processing, Newcastle, UK. 21-23 July, 2010; 827-830. ISBN-Hardcopy: 978-1-86135-370-2

37. Hershenson MB, Colin AA, Wohl ME, Stark AR. Changes in the contribution of the rib cage to tidal breathing during infancy. American Review of Respiratory Disease. 1990;141:922-5.

38. Brooks LJ, DiFiore JM, Martin RJ. Assessment of tidal volume over time in preterm infants using respiratory inductance plethysmography, The CHIME Study Group. Collaborative Home Infant Monitoring Evaluation. Pediatr Pulmonol. 1997;23:429-33. 


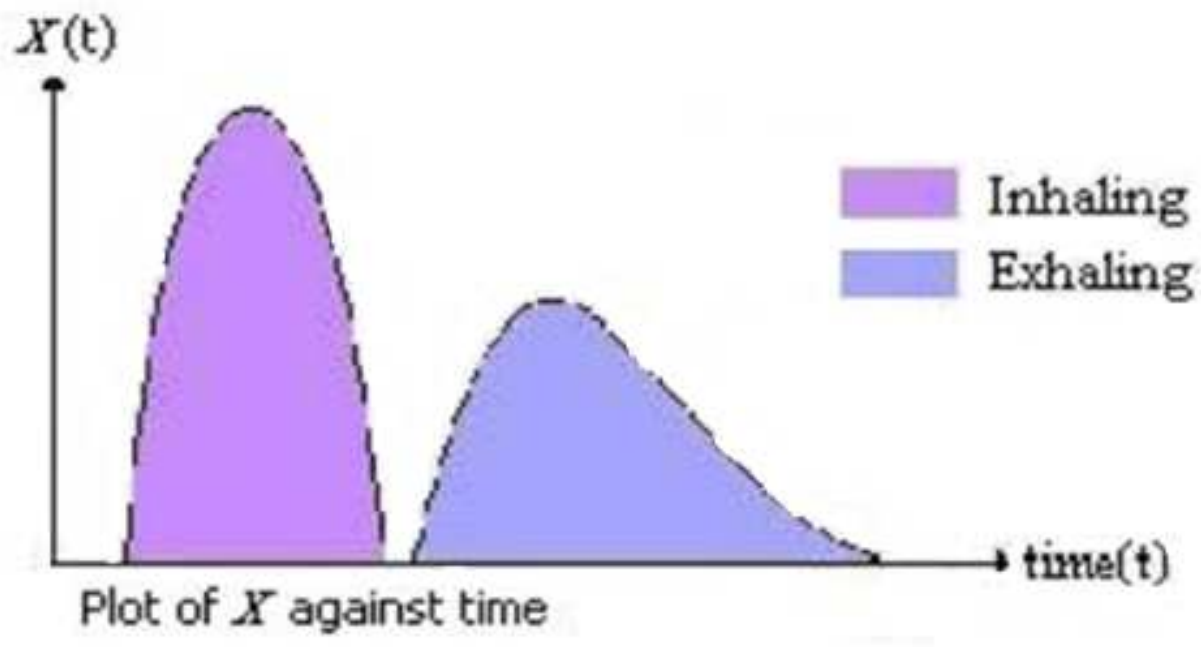

A plot of values of $x$ during inhalation and exhalation. $128 \times 68 \mathrm{~mm}(96 \times 96 \mathrm{DPI})$

John Wiley \& Sons, Inc. 


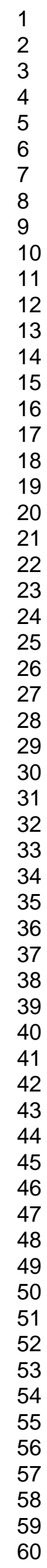

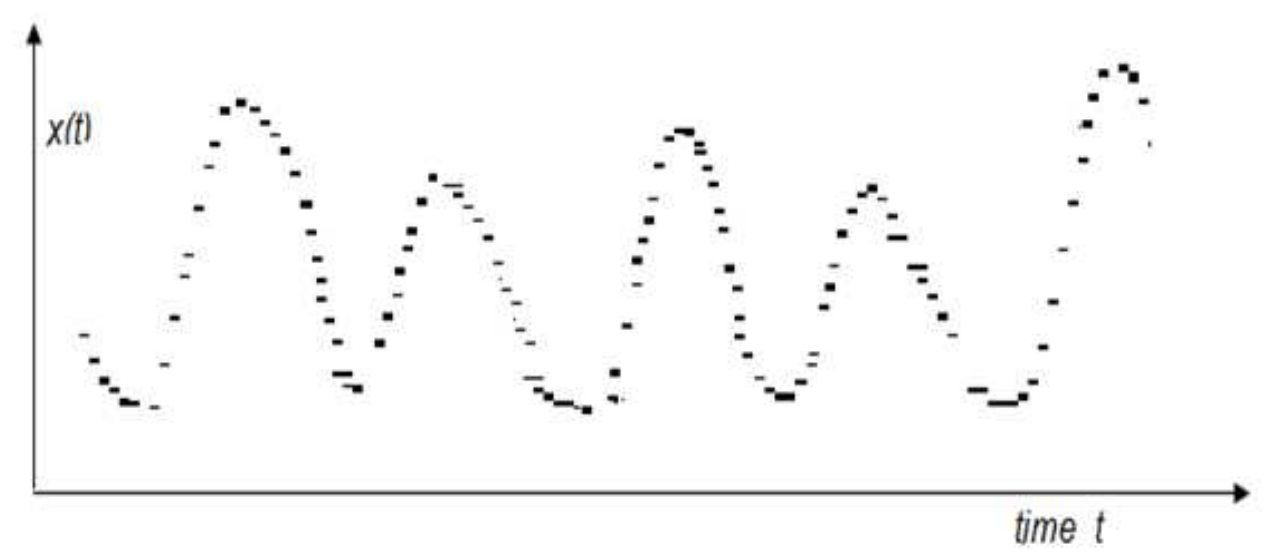

Plot $x(t)$ against time during three respiration cycles. $154 \times 69 \mathrm{~mm}(96 \times 96 \mathrm{DPI})$

John Wiley \& Sons, Inc. 
(a) A thermal image with tip of the nose represented by a circle, (b) the eight segments of the selected respiration region. $110 \times 65 \mathrm{~mm}(96 \times 96 \mathrm{DPI})$ 


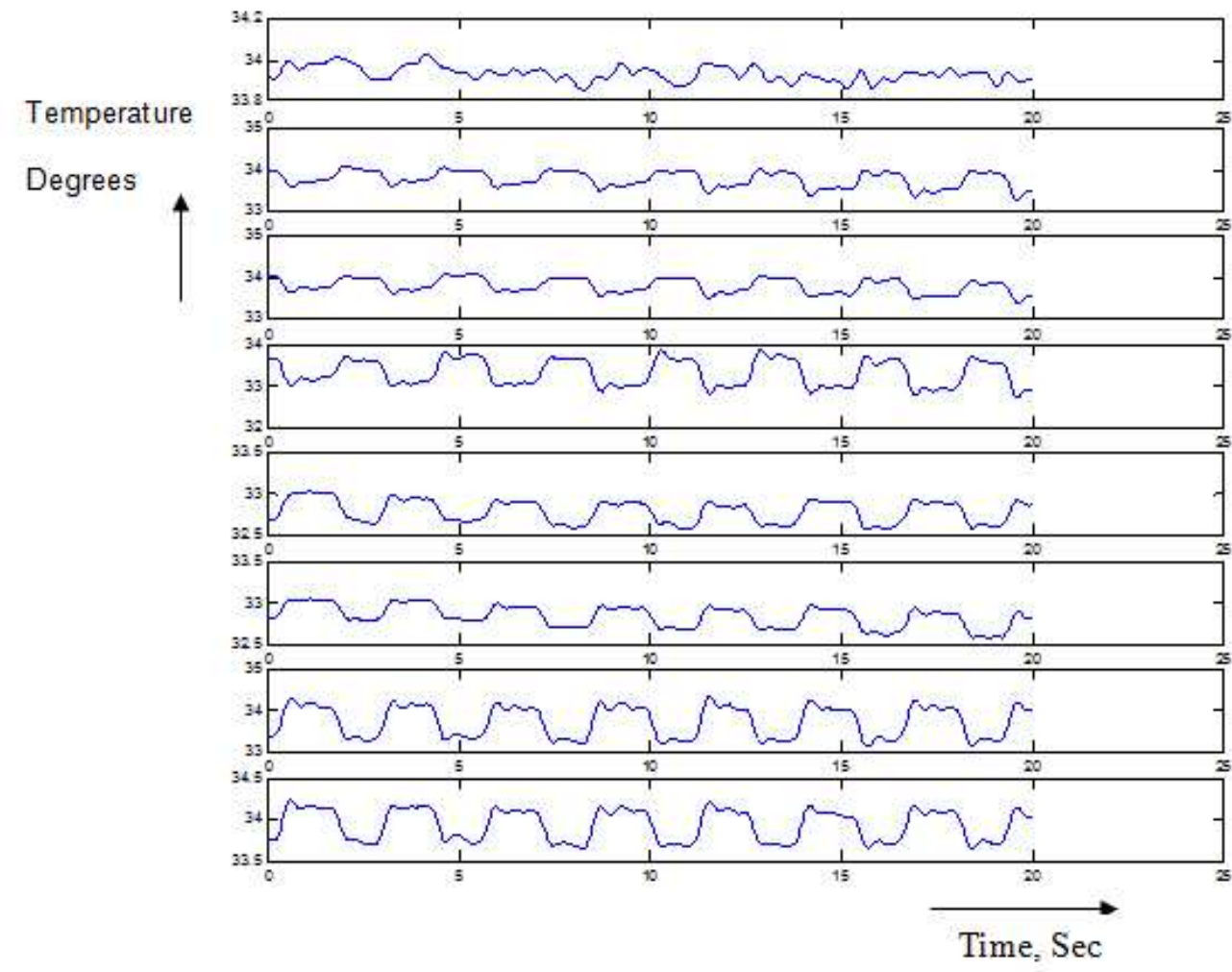

Respiration signals obtained from segments 1 to 8 (from top to bottom respectively). $148 \times 116 \mathrm{~mm}(96 \times 96 \mathrm{DPI})$

John Wiley \& Sons, Inc. 